\title{
Spectroscopy and Chemical Dynamics
}

\author{
John P. Maier* and Stefan Willitsch*
}

\begin{abstract}
Chemical physics and molecular spectroscopy are topics with a traditionally strong emphasis in the department. Current research focuses on the spectroscopic characterization of transient molecules present in interstellar space and the preparation, control and chemistry of ultracold molecules and ions in the gas phase.
\end{abstract}

Keywords: Astrophysics · Cold chemistry · Cold molecules · Interstellar molecules · Laser spectroscopy

\section{Spectroscopy of Interstellar Molecules}

\section{John P. Maier}

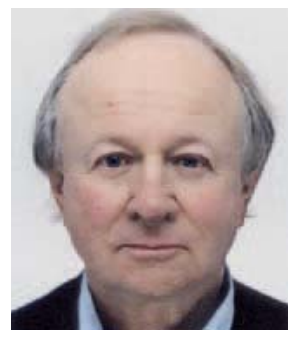

In order to learn about the structure of transient species, both neutral and ionic, so that they can be identified by spectroscopy, a number of approaches have been developed to detect and identify their electronic transitions for the first time. This is fundamental research but the results have important applications in terrestrial and astronomical environments.

Of particular interest to the research group of John P. Maier ${ }^{[1]}$ have been carbon-containing chains and rings. These are of relevance to regions of interstellar spacediffuse clouds, circumstellar shells, comets and atmospheres of planets. On earth they are intermediates in chemical reactions, flames and discharges. Once the electronic spectra of these species are known, they can be monitored there, and the details of the transitions provide information on the physical conditions of the media, such as temperature, pressure. There are three main obstacles to the detection and identification of the electronic spectra; their production in copious amount from precursors, the availability of sensitive methods for detection and the knowledge of the expected wavelength range of the transition. Because of the transient nature of the species, the most efficient means to generate them has proven to be the use of supersonic expansions coupled with discharges or laser vaporization. This enables a variety of exotic radicals to be formed under controlled conditions collision free, at very low temperatures as is pertinent for interstellar space $<100 \mathrm{~K}$, and in a molecular beam. The concentrations thus formed $\left(10^{8} \mathrm{~cm}^{-3}\right)$ are sufficient for their detection by sensitive laser-based techniques. A number of approaches are used, ranging from the use of resonance enhanced two photon-ionisation (REMPI) for the neutrals to degenerate four-wave mixing (D4WM) and cavity ring-down (CRD) for both neutrals and ions, as well as radiofrequency (RF) traps for cations. Prior to the gas phase experiments, it is an advantage to locate the electronic transitions in $6 \mathrm{~K}$ neon matrices. This is based on the implantation of mass-selected species in the inert surroundings of the neon atoms.

The following illustrates the methodology and information forthcoming. A method has been developed and used with great success to locate the electronic transitions of mass-selected species in neon matrices. This is a combination of mass spectrometry and matrix isolation techniques. The instrument built is hitherto unique worldwide (Fig 1). Specially designed sources produce the cations or anions of interest with currents around nA. Following mass selection a neon matrix is grown, a $100-200 \mu \mathrm{m}$ thin film on $1 \mathrm{~cm}^{2}$ sapphire surface at $6 \mathrm{~K}$. The concentration of the mass selected ions, or the corresponding neutrals produced by subsequent neutralization, is adequate to measure the absorption spectrum by passing light through the thin wedge of the matrix and propagating the light over $1 \mathrm{~cm}$ in a waveguide manner. Detection is via a CCD camera.
Correspondence: Prof. J. P. Maier* Department of Chemistry

University of Basel

Klingelbergstrasse 80

$\mathrm{CH}-4056$ Basel

E-mail: j.p.maier@unibas.ch

Prof. S. Willitsch*

Department of Chemistry

University of Basel

Klingelbergstrasse 80

$\mathrm{CH}-4056$ Basel

E-mail: stefan.willitsch@unibas.ch

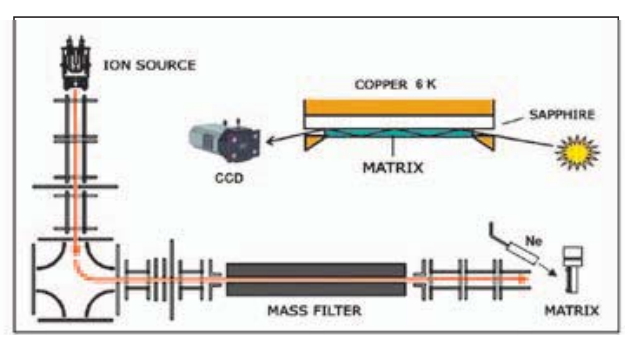

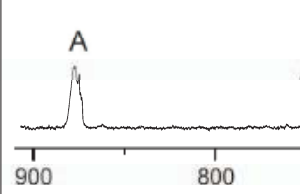
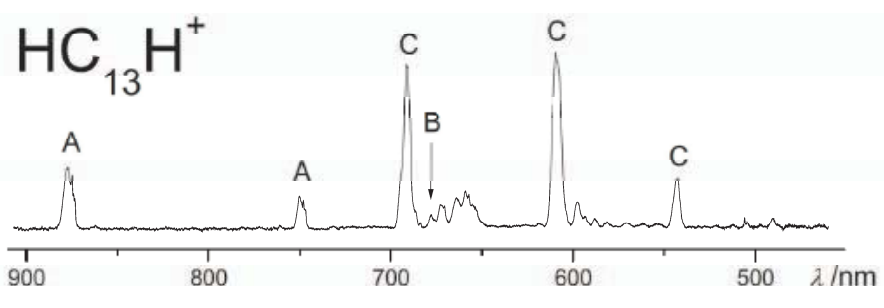

Fig. 1. Experimental set-up for the measurement of the electronic absorption spectra of massselected molecules in $6 \mathrm{~K}$ neon matrices. The inset shows the observed spectrum of $\mathrm{HC}_{13} \mathrm{H}$ obtained by codeposition of $\mathrm{HC}_{13} \mathrm{H}^{+}$ cations, generated from acetylene, with excess of neon, followed by neutralization of the ions in the matrix. 


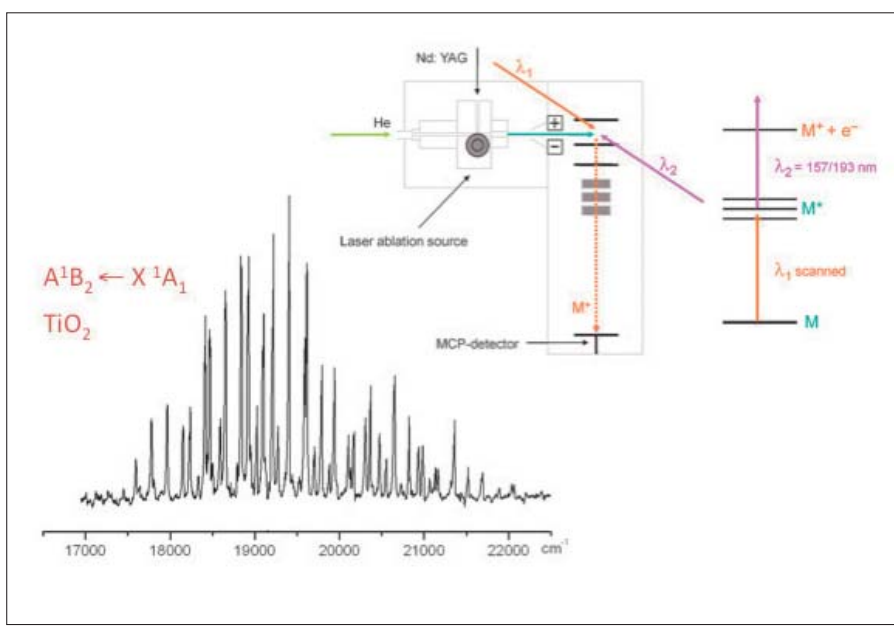

Fig. 2. Measurement of the electronic spectra of mass-selected neutral radicals using resonance enhanced ionisation technique. The spectrum shown is the $A^{1} B_{2} \leftarrow X^{1} A_{1}$ transition of $\mathrm{TiO}_{2}$, bent molecule with $C_{2 v}$ symmetry, with a complex vibrational pattern. $\mathrm{TiO}_{2}$ was 'synthesized' within a supersonic molecular beam at $50 \mathrm{~K}$ by laser ablation of Ti over which oxygen seeded in helium is passed.

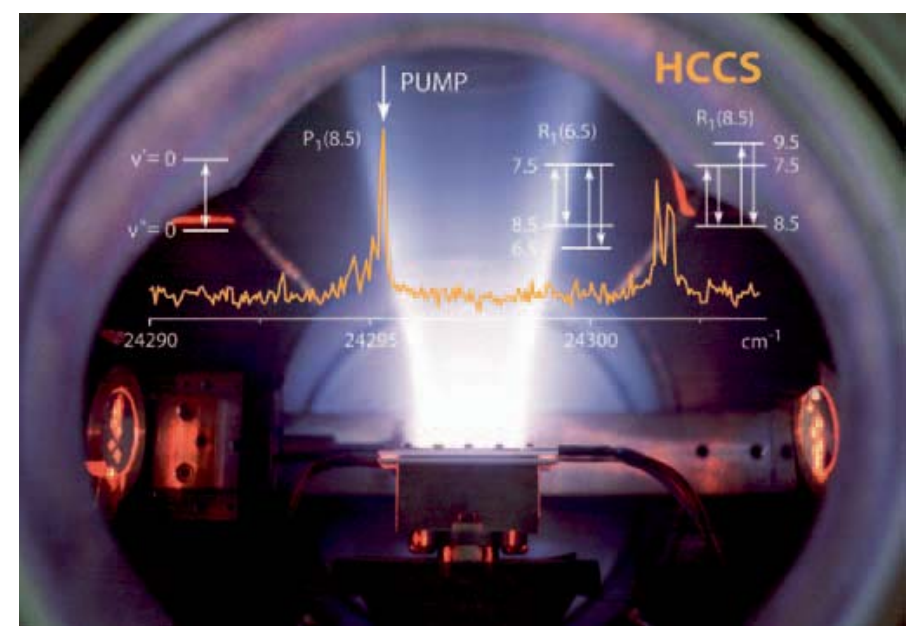

Fig. 3. Degenerate four wave mixing is a means to measure the electron spectra of transient species. The example shown is for $\mathrm{HC}_{2} \mathrm{~S}$, produced at low temperatures in a supersonic slit jet expansion of $\mathrm{CS}_{2} / \mathrm{H}_{2}$ seeded in helium, through which a discharge runs. A two colour variant of the method is used in a double resonance mode to assign the rotational lines unambiguously.
In Fig. 1 the set-up is outlined. Acetylene gas in such ion sources provides numerous species of interest; for example the polyacetylene chains, 'synthesized' in situ by radical and ion-molecule reactions. Such chains are predicted to occur in the atmosphere of Titan, a moon of Saturn with physical conditions as on early earth, in pre-oxygen days. Knowledge of their electronic spectra is a means to identify them remotely by telescopes. The absorption spectrum shown in Fig. 1 is that of neutral $\mathrm{HC}_{13} \mathrm{H}$. It is produced from the mass-selected cation trapped by releasing electrons in the neon matrix. The species is linear according to the spectroscopic pattern observed and is assigned to the A ${ }^{2} \Pi-X{ }^{2} \Pi$ transition. One characteristic is the linear dependence of the transition wavelength versus length of the molecule. The surrounding neon atoms eliminate rotation of the molecule and the $6 \mathrm{~K}$ temperature ensures that whole population is in the lowest vibrational level in the ground state. The vibrational patterns observed correspond to levels in the excited electronic state accessible by selection rules and intensities governed by Franck-Condon factors. Apart from the solvation shift the pattern mimics the absorption expected in diffuse interstellar clouds at such low temperatures. The wavelength position of the observed bands will lie within $100 \mathrm{~cm}^{-1}$ or so of their gas phase values. This alleviates the gas phase search and $\mathrm{HC}_{13} \mathrm{H}$ has in fact been observed in the gas phase using a REMPI method outlined next. In this the polyacetylenes are produced in a pulsed supersonic beam of acetylene seeded in helium through which a discharge runs. A tuneable laser excites the transition and a second laser at $157 \mathrm{~nm}$ ionises the species.
The ions are selected according to their mass and counted. Fig. 2 shows the experimental arrangement and application to obtain an electronic transition of $\mathrm{TiO}_{2}$. This molecule was studied because of its importance in terrestrial processes, catalysis, and presence in certain stars.

The species was produced in this case by laser vaporization of a titanium rod over which oxygen flows. The transition is between the ground and first excited electronic states, assigned to $\mathrm{A}^{1} \mathrm{~B}_{2} \leftarrow \mathrm{X}^{1} \mathrm{~A}_{1}$ symmetry for the inferred $\mathrm{C}_{2 \mathrm{~V}}$ structure. Under the experimental conditions used the internal temperature is around $50 \mathrm{~K}$. The spectrum shows a complex vibrational pattern, indicative of a significant geometry change in the OTiO bond angle. Increasing the resolution with a continuous wave laser enables the rotational structure to be discernible revealing a bent OTiO configuration. Hence, the combination of mass selection and spectroscopy unambiguously identifies the molecule as $\mathrm{TiO}_{2}$ and yields information on its geometry.

This is illustrated in Fig. 3 where the astrophysically interesting radical $\mathrm{HC}_{2} \mathrm{~S}$ has been probed both by CRD and D4WM methods. A slit jet expansion/discharge is used and thus the interrogating laser beam samples the radicals along a few $\mathrm{cm}$. The concentrations of radicals down to $10^{8} \mathrm{~cm}^{-3}$ can be detected. D4WM, being a non-linear technique, can be used advantageously to discriminate against overlapping absorptions and using it in a two photon colour mode as a double resonance technique to confirm assignments, as the insets of Fig. 3 indicate. The ns time resolution is better than that of CRD $(\mu \mathrm{s}$ range) and thus more sensitive to production rates of the species being synthesized in the discharge.
A new development has been the use of RF traps to measure the electronic spectra of mass-selected cations. Fig. 4 shows how this is done in the case of cyanogen cation. The latter is present in Titan's ionosphere and thus the ability to monitor it by optical spectroscopy is of relevance. The cation is produced in a conventional electron impact source and is then led via a hexapole guide and a mass-selecting quadrupole into a 22-pole ion trap. There $10^{4}$ ions are stored by a RF field. The vibrational and rotational degrees of freedom are relaxed to low temperatures by collisions with cryogenically cooled helium atoms. Detection of the electronic transition is accomplished by a two colour two photon process, analogous to the REMPI scheme used for neutrals. A tuneable laser induces the sought-after absorption whereas a second, higher energy, photon induces fragmentation, $\mathrm{CN}^{+}$in the case of $\mathrm{NCCN}^{+}$. The trap is subsequently opened, $\mathrm{CN}^{+}$is mass-selected by the further quadrupole, sent to a particle detector and counted as function of the tuneable laser wavelength.

A distinct electronic absorption system is detected (Fig. 4). The spectrum can be directly compared with astronomical observations; the temperature of the ions is around $20 \mathrm{~K}$ and the transitions observed are from the $\mathrm{v}=0$ level of the electronic ground state, $X^{2} \Pi$, to the accessible vibrational levels in the upper state, $\mathrm{B}^{2} \Sigma^{+}$. The rotational lines are not resolved because of lifetime shortening in the upper electronic state as result of intramolecular processes. This is an often encountered feature with polyatomic radicals and ions and is of significance to astronomical observations. A number of ionic systems have thus been studied, including the long linear polyacetylene cations up to $\mathrm{HC}_{18} \mathrm{H}^{+}$. 


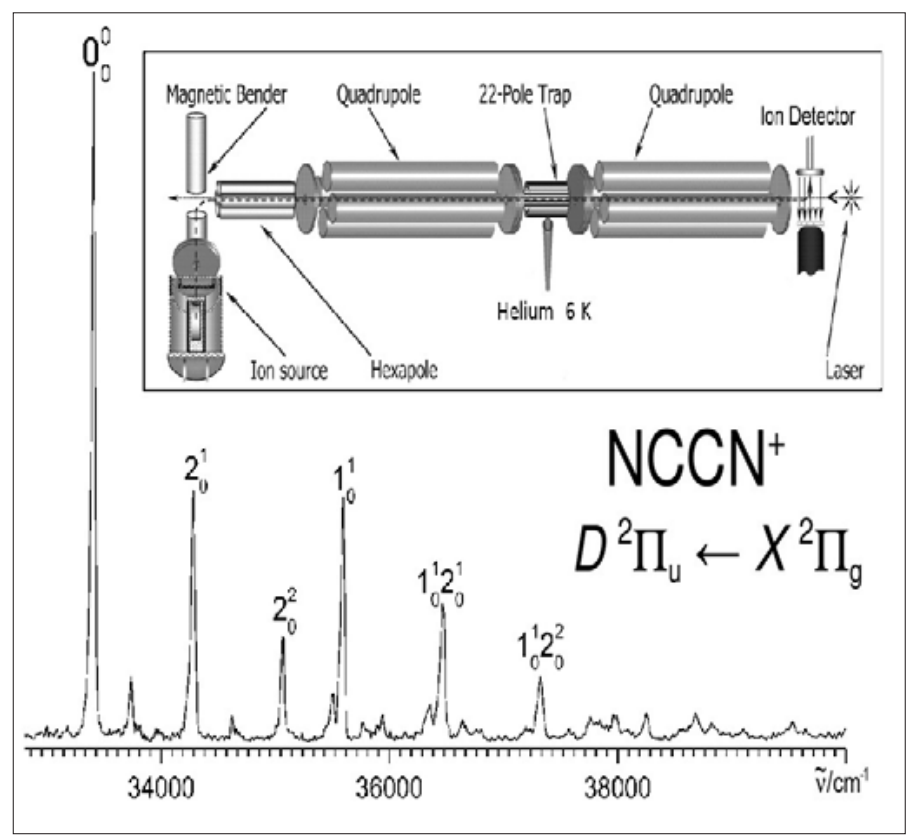

Fig. 4. Apparatus used to measure the electronic absorption of massselected cations at low temperatures. The spectrum shown is of cyanogen cation, formed in the ion source, mass-selected and stored in a 22-pole RF trap. The internal degrees of freedom are equilibrated to $20 \mathrm{~K}$ by collisions with cryogenically cooled helium. The transition of interest is located and induced by a tuneable laser while a second fixed frequency laser produces the $\mathrm{CN}^{+}$fragment. After opening of the trap the $\mathrm{CN}^{+}$ions are mass-selected and monitored. The spectrum shows the vibrationally resolved $\mathrm{B}^{2} \Sigma^{+} \leftarrow \mathrm{X}^{2} \Pi$ electronic transition.

\section{Acknowledgement}

The research of John P. Maier outlined here has only been possible because of the continuous support of the Swiss National Science Foundation over many years (current project 200020-124349/1).

\section{Chemical Dynamics at Sub-Kelvin Temperatures}

\section{Stefan Willitsch}

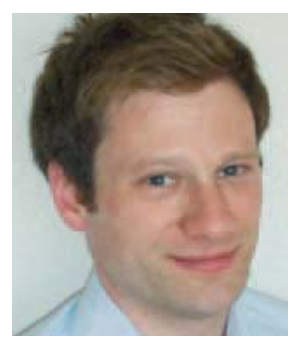

Over the past 15 years a range of methods have been developed to manipulate and cool the translational motion of molecules and molecular ions in the gas phase.[1] 'Cold' molecules at translational temperatures $T$ $<1 \mathrm{~K}$ offer a range of intriguing scientific perspectives: Temperatures as low as a few hundred nanokelvin have been reached in diatomic molecules synthesized from ultracold atoms, ${ }^{[2]}$ cold molecules have been prepared in well-defined rotational-vibrational and even hyperfine quantum states, ${ }^{[3]}$ fully energy-controlled collisional studies have been performed with velocity-controlled molecular beams ${ }^{[4]}$ and chemical reactions with single localized ions have been realized. ${ }^{[1 a, b]}$ The experimental technologies developed in this context are of considerable
[1] E. Jochnowitz, J. P. Maier, Mol. Phys. 2008, 106, 2093; R. Nagarajan, J. P. Maier, Rev. Phys. Chem. 2010, 29, 521. by thermal averaging over a broad distribution of collision energies and quantum-state populations. Consequently, cold-chemistry studies offer unique opportunities to characterize the dynamical details of chemical reactions which are not only of relevance at low temperature, but also for the understanding of chemical reactivity in general. Moreover, the tools developed for the manipulation of the motion and quantum state of molecules pave the way for schemes to accurately control chemical processes on a quantum-mechanical level.[6]

Over the past years, the research group of Stefan Willitsch has developed new approaches to study ion-molecule reactions at sub-Kelvin temperatures by combining for the first time techniques to prepare cold ions and cold neutral molecules in the same apparatus. ${ }^{[9]}$ Atomic ions such as $\mathrm{Ca}^{+}$can be cooled down to millikelvin temperatures using laser cooling. ${ }^{[1 a]}$ When confined in an ion trap, cold ions localize in space as a consequence of the balance between their mutual Coulomb repulsion and the electrical forces of the trap. The resulting regular structures of ions which are conventionally termed 'Coulomb crystals' can be observed by imaging the fluorescence of the ions generated during laser cooling (Fig. 1). By coupling a Coulombcrystal apparatus with a velocity selector to prepare continuous beams of translationally cold neutral molecules, it became possible to study ion-molecule reactions in the cold regime with extremely high sensitivity. ${ }^{[1 a, b]}$ Performing experiments with single localized ions enabled the study of reactive processes on the single-particle level and to accurately control the collision energies of the reaction partners. ${ }^{[9]}$ ] For prototypical ion-molecule reactions such as $\mathrm{Ca}^{+}+\mathrm{CH}_{3} \mathrm{~F}$, these studies demonstrated the importance of shallow submerged barriers for the chemical dynamics at very low temperatures. ${ }^{[9 b]}$

For over a decade, a persistent problem in the field was the preparation of translationally cold molecular ions in a well-defined internal, i.e. rovibrational, quantum state. Molecular ions are conventionally cooled 'sympathetically', i.e. by exchanging kinetic energy with simultaneously trapped laser-cooled atomic ions via the Coulomb interaction. Sympathetic cooling, however, does not affect the internal molecular degrees of freedom. As a consequence translationally cold, but internally 'warm' samples of ions are obtained. Fully quantum-state selected ensembles of ions, however, are essential for a range of different experiments, not only for studying the influence of the quantum state on the chemical reactivity, but also for applications in a completely different context, e.g. singleion precision spectroscopy[10] or quantuminformation processing. ${ }^{[11]}$ 


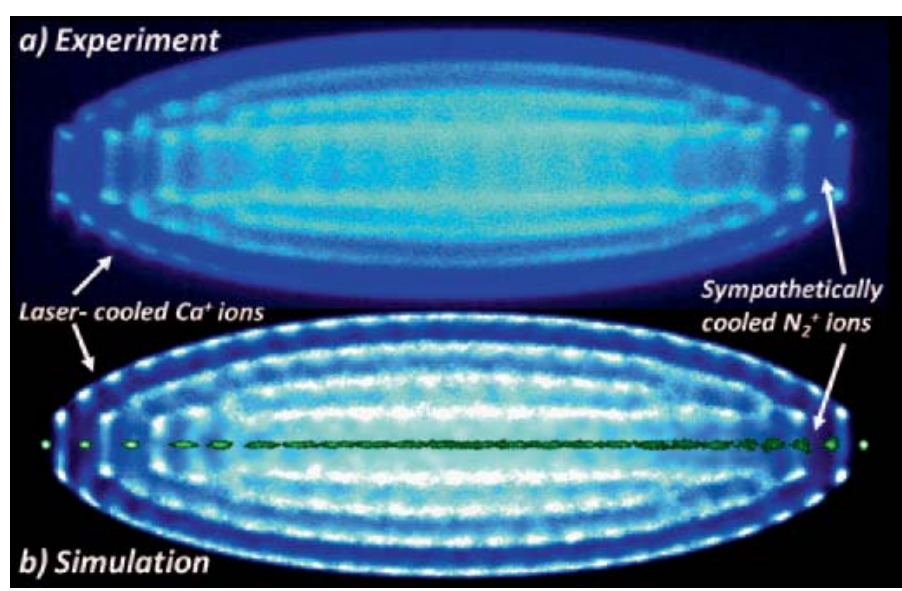
and simulated (b) falsecolour fluorescence images of a Coulomb crystal containing 925 laser-cooled $\mathrm{Ca}^{+}$ions and $24 \mathrm{~N}_{2}{ }^{+}$ions at a temperature of $15 \mathrm{mK}$. The molecular ions do not fluoresce and have been artificially made visible in the simulated image (see text).
Fig. 1. Experimental (a)

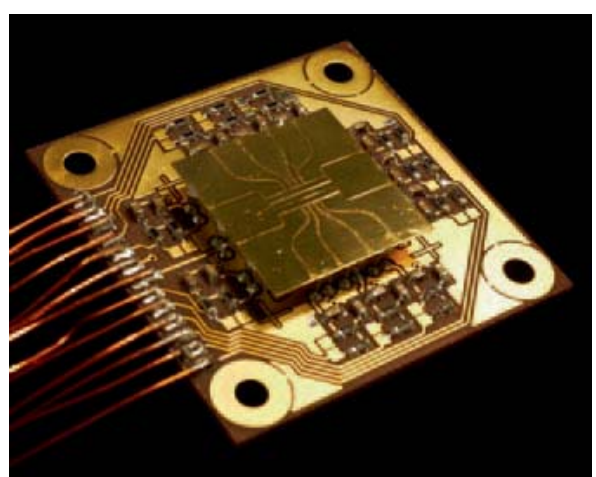

Fig. 2. A cold-chemistry 'lab-on-a-chip': Surface-electrode ion trap for the sympathetic cooling of molecular ions.

Experiments in this direction are currently in progress. lision experiments affording unprecedented control over both the collision energy and the quantum states of the reactands. As a first step an ion trap for the sympathetic cooling of molecular ions is combined with a magneto-optical trap (MOT) for the lasercooling of neutral atoms to study electrontransfer processes in the cold regime. Subsequently, a generalization of this approach is envisaged by merging ion traps with neutral-molecule traps.

Further, new ion-trap architectures are developed in the Willitsch group that provide increased flexibility as compared to the conventionally used linear Paul traps. ${ }^{[1 \mathrm{a}, \mathrm{b}]}$ In surface-electrode (SE) ion traps, ${ }^{[16]}$ all electrodes are projected onto a plane so that the ions are trapped above a surface. SE traps have thus far mainly been used for the laser-cooling of atomic ions in quantum-information experiments. ${ }^{[16]}$ However they also offer considerable advantages for chemical experiments with molecular ions. One advantage of this type of traps is the open geometry enabling improved access the trapping region and facilitating the introduction of molecular and laser beams as well as the combination with traps for neutral species. ${ }^{[15]}$ Moreover, by miniaturizing the assembly on a microchip ion-trap networks can be realized in which several connected trapping regions are implemented on a single surface. ${ }^{[16]}$ One can envisage a SE trap network in which consecutive chemical transformations and analytical tasks such as spectroscopy and mass spectrometry are performed in different trapping regions thus forming a cold-chemistry 'lab on a chip'.

Fig. 2 shows a photograph of prototype SE trap for the sympathetic cooling of molecular ions. Theoretical modelling of the trapping potentials and MD simulations of the Coulomb crystals show that sympathetically-cooled ions in such a device can be expected to exhibit significantly different thermal and structural properties compared to linear Paul traps. These properties can be adjusted by applying static voltages to control electrodes around the trapping region. ${ }^{[17]}$

\section{Acknowledgement}

The research projects of Stefan Willitsch are supported by the Swiss National Science Foundation (grant no. PP0022 118921), the NCCR Nanoscale Science and the University of Basel.

[1] a) S. Willitsch, M. T. Bell, A. Gingell, T. P. Softley, Phys. Chem. Chem. Phys. 2008, 10, 7200; b) S. Willitsch, Chimia 2009, 63, 54; c) L. D. Carr, D. DeMille, R. V. Krems, J. Ye, New J. Phys. 2009, 11, 055049; d) M. Schnell, G. Meijer, Angew. Chem. Int. Ed. 2009, 48, 6010.

[2] S. Knoop, F. Ferlaino, M. Berninger, M. Mark, H.-C. Nägerl, R. Grimm, J. P. D'Incao, B. D Esry, Phys. Rev. Lett. 2010, 104, 053201.

[3] S. Ospelkaus, K.-K. Ni, G. Quemener, B. Neyenhuis, D. Wang, M. H. G. de Miranda, J. L. Bohn, J. Ye, D. S. Jin, Phys. Rev. Lett. 2010, 104, 030402.

[4] J. J. Gilijamse, S. Hoekstra, S. Y. T. m. d. Meerakker, G. C. Groenenboom, G. Meijer, Science 2006, 313, 1617.

[5] D. C. Clary, Chem. Phys. Lett. 1995, 232, 267.

[6] R. V. Krems, Phys. Chem. Chem. Phys. 2008, 10, 4079.

[7] K.-K. Ni, S. Ospelkaus, D. Wang, G. Quemener, B. Neyenhuis, M. H. G. de Miranda, J. L. Bohn, J. Ye, D. S. Jin, Nature 2010, 464, 1324.

[8] P. F. Weck, N. Balakrishnan, Int. Rev. Phys. Chem. 2006, 25, 283. Procter, T. P. Softley, Phys. Rev. Lett. 2008, 100, 043203; b) M. Bell, A. Gingell, J. Oldham, T. P. Softley, S. Willitsch, Faraday Discuss. 2009, 142,73 .

[10] S. Schiller, V. Korobov, Phys. Rev. A 2005, 71, 032505.

[11] P. O. Schmidt, T. Rosenband, J. C. J. Koelemeij, D. B. Hume, W. M. Itano, J. C. Bergquist, D. J. Wineland, in 'AIP Conference Proceedings, Vol. CP862', Eds M. Drewsen, U. Uggerhøj, H. Knudsen, American Institute of Physics, 2006, p. 305.

[12] a) P. F. Staanum, K. Højbjerre, P. S. Skyt, A. K. Hansen, M. Drewsen, Nat. Phys. 2010, 6, 271; b) T. Schneider, B. Roth, H. Duncker, I. Ernsting, S. Schiller, Nat. Phys. 2010, 6, 275.

[13] X. Tong, A. H. Winney, S. Willitsch, Phys. Rev. Lett. 2010, 105, 143001.

[14] S. Willitsch, F. Merkt, Int. J. Mass Spectrom. 2005, 245, 14

[15] A. T. Grier, M. Cetina, F. Orucevic, V. Vuletic, Phys. Rev. Lett. 2009, 102, 223201.

[16] J. M. Amini, J. Britton, D. Leibfried, D. J. Wineland, arXiv 0812.3907.

[17] I. M. Georgescu, S. Willitsch, in preparation.

Received: October 11, 2010
[9] a) S. Willitsch, M. T. Bell, A. Gingell, S. R. to prepare state-selected and translationally cold molecular ions, the Willitsch group 